\title{
THE NOETHERIAN PROPERTY FOR QUOTIENT RINGS OF INFINITE POLYNOMIAL RINGS
}

\author{
ROBERT GILMER ${ }^{1}$ AND WILLIAM HEINZER ${ }^{2}$
}

\begin{abstract}
Let $X$ be an infinite set of indeterminates over the commutative Noetherian ring $R$ with identity. We prove that the quotient ring of $R[X]$ with respect to the multiplicative system of polynomials of unit content is also Noetherian. Moreover, we show that certain quotient rings of $R[X]$ with respect to multiplicative systems of monic polynomials (where "monic" is appropriately defined) are also Noetherian.
\end{abstract}

Let $X=\left\{X_{\lambda}\right\}_{\lambda \in \Lambda}$ be a set of indeterminates over $R$, a commutative ring with identity. The content of a polynomial $f \in R[X]$ is denoted by $C(f)$ and is defined to be the ideal of $R$ generated by the coefficients of $f$; if $C(f)=R$, then $f$ has unit content. The quotient ring of $R[X]$ with respect to the multiplicative set of polynomials of unit content is denoted by $R(X)$. Ray Heitmann has asked ${ }^{3}$ if $R(X)$ is Noetherian, provided the ring $R$ is Noetherian. We show in Theorem 6 that the answer to this question is affirmative. We subsequently define other quotient rings of $R[X]$, contained in $R(X)$ and defined in terms of certain multiplicative sets of "monic" polynomials of $R[X]$. We show the Noetherian property to be preserved also for these quotient rings of $R[X]$.

The ring $R(X)$ seems to have been first considered by Krull in [5], while Nagata introduced the notation $R(X)$ in [10, p. 17]. The following result, which is a partial citation of Proposition 33.1 of [2], lists some basic properties of the ring $R(X)$.

Proposition 1. Let $S$ be the set of elements of $R[X]$ of unit content, and let $\left\{M_{\beta}\right\}_{\beta \in B}$ be the set of maximal ideals of $R$. For each $\beta \in B$, denote by $M_{\beta}[X]$ and $M_{\beta}(X)$ the ideals of $R[X]$ and $R(X)$, respectively, generated by $M_{\beta}$.

(1) $S=R[X]-\left(\cup_{\beta \in B} M_{\beta}[X]\right)$.

(2) $\left\{M_{\beta}[X]\right\}$ is the family of ideals of $R[X]$ maximal with respect to the property of failure to meet the multiplicative system $S$; hence $\left\{M_{\beta}(X)\right\}$ is the set of maximal ideals of $R(X)$.

(3) If $Q$ is an ideal of $R$, then $Q R(X) \cap R=Q$; if $Q$ is $P$-primary in $R$, then $Q R(X)$ is $P R(X)$-primary.

Presented to the Society November 12, 1977; received by the editors September 9, 1978. AMS (MOS) subject classifications (1970). Primary 13B25, 13E05; Secondary 13C15.

Key words and phrases. Noetherian ring, polynomial of unit content, monic polynomial.

${ }^{1}$ Research supported by NSF Grant MCS 75-06591.

${ }^{2}$ Research supported by NSF Grant 7800798.

${ }^{3}$ Private communication. 
For a prime ideal $P$ of a commutative ring, we use $h(P)$ to denote the height of $P([2$, p. 109], [10, p. 24]).

LEMMA 2. If $R$ is Noetherian, if $T$ is a quotient ring of the polynomial ring $R\left[\left\{X_{\lambda}\right\}\right]$, and $P$ is a prime ideal of $T$, then $h(P) \geqslant h(P \cap R)$, and equality holds if and only if $P=(P \cap R) T .^{4}$

Proof. Since prime ideals of $T$ are the extensions of their contractions in $R\left[\left\{X_{\lambda}\right\}\right]$, it suffices to prove the result for $T=R\left[\left\{X_{\lambda}\right\}\right]$. Moreover, since prime ideals extend to prime ideals and contract to themselves with respect to polynomial ring extension, and since $R\left[\left\{X_{\lambda}\right\}\right]$ is the direct union of the polynomial rings over $R$ on finite subsets of $\left\{X_{\lambda}\right\}$, it suffices to prove the result for $T=R\left[X_{1}, \ldots, X_{n}\right]$, and hence just for the case of a polynomial ring in one variable over a Noetherian ring. The result in this case is well known ([6, §6], [4, Theorem 149]).

LEMMA 3. If $R$ is Noetherian, if $T$ is a quotient ring of the polynomial ring $R\left[\left\{X_{\lambda}\right\}\right]$, and $P$ is a prime ideal of $T$ of finite height, then there exists a finite subset $\left\{X_{i}\right\}_{i=1}^{n}$ of $\left\{X_{\lambda}\right\}$ such that $P=\left(P \cap R\left[X_{1}, \ldots, X_{n}\right]\right) T$.

Proof. Again, since each prime ideal of $T$ is the extension of its contraction to $R\left[\left\{X_{\lambda}\right\}\right]$, we may assume that $T=R\left[\left\{X_{\lambda}\right\}\right]$. If $h(P)=r$, let $P_{0}<P_{1}$ $<\cdots<P_{r}=P$ be a chain of prime ideals of $T$, and let $f_{i} \in P_{i} \backslash P_{i-1}$, $i=1, \ldots, r$. The $f_{i}$ are polynomials in a finite subset $\left\{X_{i}\right\}_{i=1}^{n}$ of $\left\{X_{\lambda}\right\}$ and $h(P)=h\left(P \cap R\left[X_{1}, \ldots, X_{n}\right]\right)$. Hence, $P=\left(P \cap R\left[X_{1}, \ldots, X_{n}\right]\right) T$ by Lemma 2.

THEOREM 4. If $R$ is Noetherian, if $T$ is a quotient ring of the polynomial ring $R\left[\left\{X_{\lambda}\right\}\right]$, and $P$ is a prime ideal of $T$ of finite height, then $P$ is finitely generated.

Proof. By Lemma 3, $P=\left(P \cap R\left[X_{1}, \ldots, X_{n}\right]\right) T$. Since $R$ is Noetherian, $R\left[X_{1}, \ldots, X_{n}\right]$ is Noetherian by the Hilbert Basis Theorem. Hence $P \cap$ $R\left[X_{1}, \ldots, X_{n}\right]$, and therefore $P$, is finitely generated.

THEOREM 5. If $R$ is Noetherian and $T$ is a quotient ring of the polynomial ring $R\left[\left\{X_{\lambda}\right\}\right]$, then $T$ is Noetherian if and only if each prime ideal of $T$ has finite height.

Proof. By Krull's Principal Ideal Theorem, each prime ideal of a Noetherian ring has finite height. Therefore the condition is clearly necessary. If each prime ideal of $T$ has finite height, then, by Theorem 4, each prime ideal of $T$ is finitely generated, so by a theorem of Cohen $([4$, p. 5] or $[10, p$. 8]) $T$ is Noetherian. 364].

${ }^{4}$ If $R$ is not Noetherian, it can happen that $P=(P \cap R) R[X]$ and $h(P)>h(P \cap R)[2, \mathrm{p}$. 
THEOREM 6. If $R$ is Noetherian, then $R(X)=R\left(\left\{X_{\lambda}\right\}_{\lambda \in \Lambda}\right)$ is also Noetherian.

Proof. By Theorem 5 and Proposition 1, it suffices to show for each maximal ideal $M$ of $R$ that $M R\left[\left\{X_{\lambda}\right\}\right]$ has finite height. Since $R$ is Noetherian, $M$ is of finite height, and, by Lemma $2, h(M)=h\left(M R\left[\left\{X_{\lambda}\right\}\right]\right)$.

Assume that $Y$ is an indeterminate over the ring $R$. A ring closely related to $R(Y)$ is the quotient ring of $R[Y]$ with respect to the multiplicative system of monic polynomials over $R$. We denote this ring by $R\langle Y\rangle$; it arises in Quillen's proof of the Serre Conjecture [11], and has been the object of some other recent investigation ([1], [7, Chapter IV], [8]). For a polynomial ring in two variables over $R$, say $R\left[X_{1}, X_{2}\right]$, a natural analogue of $R\langle Y\rangle$ is obtained by defining $R\left\langle X_{1}, X_{2}\right\rangle$ to be the quotient ring of $R\left\langle X_{1}\right\rangle\left[X_{2}\right]$ at the multiplicative system of monic polynomials in $X_{2}$ over $R\left\langle X_{1}\right\rangle$. By induction we define

$$
R\left\langle X_{1}, \ldots, X_{n}\right\rangle=R\left\langle X_{1}, \ldots, X_{n-1}\right\rangle\left\langle X_{n}\right\rangle \text {. }
$$

Note that the definition of $R\left\langle X_{1}, \ldots, X_{n}\right\rangle$, unlike that of $R\left(X_{1}, \ldots, X_{n}\right)$, depends upon the order of the indeterminates. For example, it is proved in [3, Proposition 10] that $R\left\langle X_{1}, X_{2}\right\rangle$ and $R\left\langle X_{2}, X_{1}\right\rangle$ are equal as subrings of the total quotient ring of $R\left[X_{1}, X_{2}\right]$ if and only if $R$ is 0 -dimensional.

In general, if $\Lambda$ is a totally ordered set and $\left\{X_{\lambda}\right\}_{\lambda \in \Lambda}$ is a set of indeterminates over $R$, then we define $R\left\langle\left\{X_{\lambda}\right\}\right\rangle$ to be the union of the directed set $\left\{R\left\langle X_{\lambda_{1}}, \ldots, X_{\lambda_{n}}\right\rangle\right\}$ of subrings of the total quotient ring of $R\left[\left\{X_{\lambda}\right\}\right]$, the union being taken over all finite subsets $\left\{\lambda_{1}<\lambda_{2}<\cdots<\lambda_{n}\right\}$ of $\Lambda$. It is clear that $R\left\langle\left\{X_{\lambda}\right\}\right\rangle$ so defined is a regular quotient $\operatorname{ring}^{6}$ of $R\left[\left\{X_{\lambda}\right\}\right]$. We proceed to show that $R\left\langle\left\{X_{\lambda}\right\}\right\rangle=R\left[\left\{X_{\lambda}\right\}\right]_{S}$, where $S$ is the multiplicative system of "monic" polynomials in $R\left[\left\{X_{\lambda}\right\}\right]$, defined in the following manner. The total order on $\Lambda$ induces, via the reverse lexicographic order, a total order on the set of monomials ${ }^{7}$ in the indeterminates $X_{\lambda}$, as follows. If $\lambda_{1}, \ldots, \lambda_{n} \in \Lambda$ are such that $\lambda_{1}<\lambda_{2}<\cdots<\lambda_{n}$ and if $e_{1}, \ldots, e_{n}$, $f_{1}, \ldots, f_{n}$ are nonnegative integers, then

$$
X_{\lambda_{1}}^{e_{1}} X_{\lambda_{2}}^{e_{2}} \cdots X_{\lambda_{n}}^{e_{n}}<X_{\lambda_{1}}^{f_{1}} X_{\lambda_{2}}^{f_{2}} \cdots X_{\lambda_{n}}^{f_{n}}
$$

if and only if $e_{i}<f_{i}$ for the largest integer $i$ such that the corresponding exponents are unequal. This order on monomials is compatible with multiplication. If $g$ is a nonzero element of $R\left[\left\{X_{\lambda}\right\}\right]$, then $g$ can be written in the form

$$
g=r_{1} Y_{1}+\cdots+r_{k} Y_{k}
$$

\footnotetext{
${ }^{5}$ Quillen uses the notation $R(Y)$ for the quotient ring of $R[Y]$ with respect to the multiplicative system of monic polynomials.

${ }^{6}$ Regularity follows from the fact that an element of $R[X]$ is a zero divisor if and only if it is annihilated by a nonzero element of $R([8],[2,(28.7)])$.

${ }^{7} \mathrm{By}$ monomial, it is to be understood here that the coefficient from $R$ is 1 .
} 
where $r_{i} \in R, r_{k} \neq 0$, and $Y_{1}, \ldots, Y_{k}$ are monomials such that $Y_{1}<\cdots<$ $Y_{k}$. We call $Y_{k}$ the leading monomial of $g, r_{k}$ the leading coefficient of $g$, and say that $g$ is monic if $r_{k}=1$. Let $S$ be the set of monic polynomials in $R\left[\left\{X_{\lambda}\right\}\right]$. It is clear that $S$ is a multiplicative system in $R\left[\left\{X_{\lambda}\right\}\right]$. Since $S \cap R\left[X_{\lambda_{1}}, \ldots, X_{\lambda_{n}}\right]$ is the set of monic polynomials in $R\left[X_{\lambda_{1}}, \ldots, X_{\lambda_{n}}\right]$, and since $R\left\langle\left\{X_{\lambda}\right\}\right\rangle$ is defined to be $\cup\left\{R\left\langle X_{\lambda_{1}}, \ldots, X_{\lambda_{n}}\right\rangle \mid\left\{\lambda_{i}\right\}_{1}^{n}\right.$ is a finite subset of $\Lambda$, it is sufficient to establish the equality $R\left\langle\left\{X_{\lambda}\right\}\right\rangle=R\left[\left\{X_{\lambda}\right\}\right]_{S}$ in the case where $\Lambda$ is finite. For the case of one variable, say $X_{1}, R\left\langle X_{1}\right\rangle=R\left[X_{1}\right]_{S}$ by definition. For $n$ variables, say $X_{1}, \ldots, X_{n}$, it is clear that $R\left[X_{1}, \ldots, X_{n}\right]_{S}$ is contained in $R\left\langle X_{1}, \ldots, X_{n}\right\rangle$, and proceeding by induction, we may assume that $R\left\langle X_{1}, \ldots, X_{n-1}\right\rangle\left[X_{n}\right]$ is contained in $R\left[X_{1}, \ldots, X_{n}\right]_{s}$. If $f$ is a polynomial in $R\left\langle X_{1}, \ldots, X_{n-1}\right\rangle\left[X_{n}\right]$ that is monic as a polynomial in $X_{n}$ with coefficients in $R\left\langle X_{1}, \ldots, X_{n-1}\right\rangle$, then we wish to show that $f$ is a unit in $R\left[X_{1}, \ldots, X_{n}\right]_{s}$. We have $f=X_{n}^{m}+a_{m-1} X_{n}^{m-1}+\cdots+a_{0}$ with the $a_{i} \in$ $R\left\langle X_{1}, \ldots, X_{n-1}\right\rangle$. By the induction hypothesis, there exists $s \in S \cap$ $R\left[X_{1}, \ldots, X_{n-1}\right]$ such that $s a_{i} \in R\left[X_{1}, \ldots, X_{n-1}\right]$ for $i=1, \ldots, m-1$. If $s$ has leading monomial $Y$, then $Y X_{n}^{m}$ is the leading monomial of $s f \in$ $R\left[X_{1}, \ldots, X_{n}\right]$, and $s f$ is a monic polynomial in $R\left[X_{1}, \ldots, X_{n}\right]$. Therefore sf $\in S$ and $f$ is a unit of $R\left[X_{1}, \ldots, X_{n}\right]$. We conclude that $R\left\langle\left\{X_{\lambda}\right\}\right\rangle=$ $R\left[\left\{X_{\lambda}\right\}\right]_{S}$.

We shall prove in Theorem 9 that the ring $R\left\langle\left\{X_{\lambda}\right\}\right\rangle$ is Noetherian if $R$ is Noetherian; for finite dimensional $R$, this follows readily from Theorem 5 .

Proposition 7. Let $\Lambda$ be a totally ordered set and let $\left\{X_{\lambda} \mid \lambda \in \Lambda\right\}$ be a set of indeterminates over the finite dimensional Noetherian ring $R$. Then $R\left\langle\left\{X_{\lambda}\right\}_{\lambda \in \Lambda}\right\rangle$ is Noetherian.

Proof. By Theorem 5, it suffices to show that $R\left\langle\left\{X_{\lambda}\right\}\right\rangle$ is finite dimensional. If $J$ is an $n$-dimensional Noetherian ring, it is known that $J\langle Y\rangle$ again has dimension $n$ ([1], [7, Proposition 1.2, Chapter IV]). Since $R\left\langle\left\{X_{\lambda}\right\}\right\rangle$ is the direct limit of the rings $R\left\langle X_{\lambda_{1}}, \ldots, X_{\lambda_{3}}\right\rangle$ for finite subsets $\left\{X_{\lambda_{i}}\right\}_{i=1}^{s}$ of $\left\{X_{\lambda}\right\}$, and since the direct limit of rings of dimension $n$ is a ring of dimension $<n$, we conclude that $R\left\langle\left\{X_{\lambda}\right\}\right\rangle$ is Noetherian. (In fact, $\operatorname{dim} R\left\langle\left\{X_{\lambda}\right\}\right\rangle=n$.)

REMARK 8. If $N$ is a multiplicative system in $R$, then there exist canonical homomorphisms $\varphi: R(X)_{N} \rightarrow R_{N}(X)$ and $\psi: R\langle X\rangle_{N} \rightarrow R_{N}\langle X\rangle$. In general, these canonical homomorphisms are not surjective. This is the case even if $N$ is the complement of a maximal ideal of $R$. For example, if $R$ is a polynomial ring in two variables over an algebraically closed field and $N$ is the complement of a maximal ideal of $R$, then it is easy to see that $R(X)_{N}$ and $R\langle X\rangle_{N}$ are properly contained in $R_{N}(X)$ and $R_{N}\langle X\rangle$ as subrings of the quotient field of $R[X]$. Thus, to show $R\left\langle\left\{X_{\lambda}\right\}\right\rangle$ is Noetherian for $R$ an infinite dimensional Noetherian ring, something other than just a naive localization argument and an application of Proposition 7 is necessary. We show below, however, that a modified local :ation argument does work. 
THEOREM 9. If $\Lambda$ is a totally ordered set and $\left\{X_{\lambda} \mid \lambda \in \Lambda\right\}$ is a set of indeterminates over the Noetherian ring $R$, then $R\left\langle\left\{X_{\lambda}\right\}\right\rangle$ is Noetherian.

Proof. Let $S$ be the multiplicative system of monic polynomials in $R\left[\left\{X_{\lambda}\right\}\right]$. We have $R\left[\left\{X_{\lambda}\right\}\right]_{S}=R\left\langle\left\{X_{\lambda}\right\}\right\rangle$, and to show that $R\left\langle\left\{X_{\lambda}\right\}\right\rangle$ is Noetherian, it suffices, by Theorem 5 , to show that if $P$ is a prime ideal of $R\left[\left\{X_{\lambda}\right\}\right]$ not meeting $S$, then $P$ is of finite height. Consider the set $A$ consisting of zero and the set of leading coefficients of elements of $P$. It is clear that $A$ is closed under multiplication by elements of $R$. We note that $A$ is also closed under subtraction. For suppose $r_{1}$ and $r_{2}$ are the leading coefficients of $f_{1}$ and $f_{2}$ in $P$. Certainly $r_{1}-r_{2}$ is in $A$ if $r_{1}=0, r_{2}=0$, or $r_{1}=r_{2}$. Otherwise, if $m_{i}$ is the leading monomial of $f_{i}$, then $r_{1}-r_{2}$ is the leading coefficient of $f_{1} m_{2}-f_{2} m_{1} \in P$. Therefore $A$ is an ideal of $R$, and since $P$ does not meet $S$, the ideal $A$ is proper. Let $M$ be a maximal ideal of $R$ containing $A$, and let $\varphi$ denote the canonical homomorphism of $R\left[\left\{X_{\lambda}\right\}\right]$ into $R_{M}\left[\left\{X_{\lambda}\right\}\right]$. We prove that the prime ideal $\varphi(P) R_{M}\left[\left\{X_{\lambda}\right\}\right]$ does not meet the multiplicative system of monic polynomials of $R_{M}\left[\left\{X_{\lambda}\right\}\right]$. Suppose not. Then there exist $f \in P$ and $r \in R \backslash M$ such that $\varphi(f) / \varphi(r)$ is a monic polynomial in $R_{M}\left[\left\{X_{\lambda}\right\}\right]$. If $Y$ is the leading monomial of $\varphi(f) / \varphi(r)$ in $R_{M}\left[\left\{X_{\lambda}\right\}\right]$, then there exists $t \in R \backslash M$ such that $t f$ is in $R\left[\left\{X_{\lambda}\right\}\right]$ and has leading monomial $Y$. If $u$ is the leading coefficient of $t f$, then $\varphi(u)=\varphi(r t)$. Since $r t \notin M$, we have $u \notin M$. But $t f \in P$ so that $u \in A$. This contradicts the fact that $A$ is contained in $M$. Therefore $\varphi(P) R_{M}\left[\left\{X_{\lambda}\right\}\right]$ extends to a proper ideal in $R_{M}\left\langle\left\{X_{\lambda}\right\}\right\rangle$. Since $R_{M}$ is a finite dimensional Noetherian ring, $R_{M}\left\langle\left\{X_{\lambda}\right\}\right\rangle$ is Noetherian by Proposition 7. Therefore $\varphi(P) R_{M}\left[\left\{X_{\lambda}\right\}\right]$ is a prime ideal of finite height. Since $h(P)=h\left(\varphi(P) R_{M}\left[\left\{X_{\lambda}\right\}\right]\right)$, this completes the proof of Theorem 9.

If $\sigma$ is a nonidentity permutation on the set of positive integers, and $R$ is a ring of dimension greater than 0 , then it follows from [3, Proposition 10] that $R\left\langle X_{1}, X_{2}, \ldots\right\rangle$ and $R\left\langle X_{o 1}, X_{\sigma 2}, \ldots\right\rangle$ are distinct subrings of the total quotient ring of $R\left[\left\{X_{i}\right\}\right]$. Of course, these rings are $R$-isomorphic under the mapping taking $X_{i}$ to $X_{\sigma i}$, and by Theorem 9 , each is Noetherian if $R$ is Noetherian. It seems natural, therefore, to consider the ring $T=$ $\cap\left\{R\left\langle X_{\sigma 1}, X_{\sigma 2}, \ldots\right\rangle \mid \sigma\right.$ is a permutation on the set of positive integers $\}$, and the multiplicative system $S$ of $R\left[\left\{X_{i}\right\}\right]$ consisting of polynomials that are monic with respect to each ordering of the set of positive integers defined by a permutation $\sigma$. We proceed to show that $T=R\left[\left\{X_{i}\right\}\right]_{S}$, and that this ring is Noetherian if $R$ is Noetherian.

Let $G_{n}$ denote the permutation group on $\{1, \ldots, n\}$, and for $\sigma \in G_{n}$, let $N_{\sigma}$ denote the multiplicative system of monic polynomials in $R\left[X_{1}, \ldots, X_{n}\right]$ under the ordering $\sigma 1<\sigma 2<\cdots<\sigma n$. Thus $R\left[X_{1}, \ldots, X_{n}\right]_{N}=$ $R\left\langle X_{\sigma 1}, \ldots, X_{o n}\right\rangle$. Note that any monomial in $X_{1}, \ldots, X_{n}$ is in $N_{\sigma}$, and if $f$ is an element of $N_{\sigma}$ and $m$ is a moriomial, then $m f$ is again in $N_{\sigma}$. 
LEMMA 10. If $f_{\sigma}$ is an element of $N_{\sigma}$ for each $\sigma$ in $G_{n}$, then there exist monomials $m_{\sigma}$ in $X_{1}, \ldots, X_{n}$ such that $f=\sum m_{a} f_{\sigma}$ is in each $N_{\sigma}$. In particular, if $A$ is an ideal of $R\left[X_{1}, \ldots, X_{n}\right]$ meeting each of the multiplicative systems $N_{o}$, then $A$ contains an element $f$ such that $f$ is in each $N_{\sigma}$.

Proof. We proceed by induction on $n$, the case $n=1$ being immediate. Let $\sigma_{i} \in G_{n}$ be such that $\sigma_{i} n=i$ for $i=1, \ldots, n$. For a fixed $i$, consider all $\tau$ in $G_{n}$ such that $\tau n=i$. Using the induction hypothesis that the result is true for $n-1$, we obtain a linear combination with monomial coefficients of the $f_{\tau}$ such that $\tau n=i$, say $g_{i}$, that is in every $N_{\tau}$ for which $\tau n=i$. It remains to show that there exist monomials, say $p_{i}$, in $X_{1}, \ldots, X_{n}$ such that $\sum_{i=1}^{n} p_{i} g_{i}$ is in every $N_{\sigma}$. We choose $p_{i}=X_{i}^{e_{i}}$, where $e_{i}$ is a positive integer greater than the degree in $X_{i}$ of any $g_{j}$. It follows that $\sum_{i=1}^{n} X_{i}^{e_{i}} g_{i}$ is in every $N_{\sigma}$.

THEOREM 11. Let $R$ be a Noetherian ring and let $S$ be the multiplicative system in $R\left[\left\{X_{i}\right\}_{i=1}^{\infty}\right]$ of polynomials that are monic with respect to each ordering of the $X_{i}$. Then $R\left[\left\{X_{i}\right\}\right]_{S}$ is again Noetherian, and is equal to $\cap\left\{R\left\langle X_{\sigma 1}, X_{\sigma 2}, \ldots\right\rangle \mid \sigma\right.$ is a permutation on the set of positive integers $\}$.

Proof. For each permutation $\sigma$, let $S_{\sigma}$ denote the multiplicative system of polynomials of $R\left[\left\{X_{i}\right\}\right]$ that are monic with respect to the ordering $\sigma 1<\sigma 2$ $<\ldots$ Then $S=\cap_{\sigma} S_{\sigma}$, and to show that $R\left[\left\{X_{i}\right\}\right]_{S}$ is Noetherian, it suffices, by Theorem 5 , to show that each prime ideal $P$ of $R\left[\left\{X_{i}\right\}\right]$ that does not meet $S$ is of finite height. If $P$ does not meet $S_{\sigma}$ for some $\sigma$, then it is clear that $P$ is of finite height, for $R\left[\left\{X_{i}\right\}\right]_{S_{o}}=R\left\langle X_{o 1}, X_{\sigma 2}, \ldots\right\rangle$ is Noetherian by Theorem 9. Hence $P R\left\langle X_{\sigma 1}, X_{\sigma 2}, \ldots\right\rangle$, and therefore $P$, is of finite height. We show that if $P$ is a prime ideal of $R\left[\left\{X_{i}\right\}\right]$ that meets each $S_{\sigma}$, then $P$ meets $S$. Consider $R\left[X_{1}, \ldots, X_{n}\right]$ and the $n !$ multiplicative systems $N_{\tau}$ of $R\left[X_{1}, \ldots, X_{n}\right]$ associated with permutations $\tau$ of $\{1, \ldots, n\}$. For each $S_{\sigma}$ we have $S_{\sigma} \cap R\left[X_{1}, \ldots, X_{n}\right]=N_{\tau}$ for some $\tau$. If $P$ meets each of the $N_{\tau}$ on $R\left[X_{1}, \ldots, X_{n}\right]$, then, by Lemma $10, P$ contains an element $f$ such that $f$ belongs to each $N$, and therefore to each $S_{\sigma}$, and hence to $S=\cap_{\sigma} S_{\sigma}$. But, if for each positive integer $n$, there is a multiplicative system $N_{\tau_{n}}$ on $R\left[X_{1}, \ldots, X_{n}\right]$ such that $P \cap N_{\tau_{n}}$ is empty, then a standard compactness argument that the inverse limit of finite nonempty sets is nonempty yields the existence of a chain $N_{\tau_{1}} \subset N_{\tau_{2}} \subset \ldots$ of such sets. And $\cup_{n=1}^{\infty} N_{\tau_{n}}=S_{o}$ for some $\sigma$. Hence $P \cap S$ is empty. Therefore if $P$ does not meet $S$, then $P$ does not meet $S_{\sigma}$, and we have shown that $R\left[\left\{X_{i}\right\}\right]$ is Noetherian. It is clear that $R\left[\left\{X_{i}\right\}\right]_{s}$ is contained in $T=\cap R\left\langle X_{o 1}, X_{o 2}, \ldots\right\rangle$. To show that $T$ is contained in $R\left[\left\{X_{i}\right\}\right]_{S}$, take $y \in T$, and let $A=\left\{f \in R\left[\left\{X_{i}\right\}\right] \mid f y \in R\left[\left\{X_{i}\right\}\right]\right\}$. Then $A$ is an ideal of $R\left[\left\{X_{i}\right\}\right]$, and by the definition of $T$ it follows that $A$ meets each $S_{\sigma}$. By Lemma 10 and the compactness argument quoted above, $A$ meets $S$, and hence $y \in R\left[\left\{X_{i}\right\}\right]_{S}$. This completes the proof of Theorem 11 .

REMARK 12. If $\Lambda$ is any totally ordered set and $\left\{X_{\lambda} \mid \lambda \in \Lambda\right\}$ is a set of indeterminates over a Noetherian ring $R$, then in analogy with Theorem 11, 
we can consider the group $G$ of permutations of $\Lambda$. Each $g$ in $G$ induces a total order on $\Lambda$, and hence an ordering of the $X_{\lambda}$. Let $S_{g}$ denote the multiplicative system of monic polynomials of $R\left[\left\{X_{\lambda}\right\}\right]$ with respect to the ordering defined by $g$. By Theorem $9, R\left[\left\{X_{\lambda}\right\}\right]_{S_{g}}$ is Noetherian for each $g$ in $G$. Indeed, in analogy with Theorem 11, if $S=\cap\left\{S_{g} \mid g \in G\right\}$, then $R\left[\left\{X_{\lambda}\right\}\right]_{S}=\cap_{g} R\left[\left\{X_{\lambda}\right\}\right]_{S_{g}}$, and this ring is again Noetherian. The proof for this result is similar to the proof given for Theorem 11, simply making use of the fact that $R\left[\left\{X_{\lambda}\right\}\right]$ is the direct union of the polynomial rings over $R$ in a finite number of the $X_{\lambda}$, and the fact that any finite totally ordered set of cardinality $n$ has the same order structure as the natural order on the set of positive integers $\leqslant n$.

We note that for $R$ Noetherian, it follows from Theorem 5 that, in general, a quotient ring $R\left[\left\{X_{\lambda}\right\}\right]_{S}$ is Noetherian if $R[Y]_{S \cap R[Y]}$ is Noetherian for each countably infinite subset $Y$ of $\left\{X_{\lambda}\right\}$.

\section{REFERENCES}

1. J. W. Brewer and D. L. Costa, Projective modules over some non-Noetherian polynomial rings, J. Pure Appl. Algebra (to appear).

2. R. Gilmer, Multiplicative ideal theory, Marcel Dekker, New York, 1972. MR 55 \#323.

3. R. Gilmer and W. Heinzer, On the divisors of monic polynomials over a commutative ring, Pacific J. Math. 78 (1978), 121-131.

4. I. Kaplansky, Commutative rings, Allyn and Bacon, Boston, Mass., 1970. MR 40 \#234.

5. W. Krull, Beiträge zur Arithmetik kommutativer Integritätsbereiche. VII. Multiplikativ abgeschlossene Systeme von endlichen Idealen, Math. Z. 48 (1943), 533-552. MR 5, 33.

6. __ Jacobsonsche Ringe, Hilbertscher Nullstellensatz, Dimensionentheorie, Math. Z. 54 (1951), 354-387. MR 13, 903.

7. T. Y. Lam, Serre's conjecture, Lecture Notes in Math., vol. 635, Springer-Verlag, Berlin and New York, 1978.

8. R. Matsuda, Infinite group rings. III, Bull. Fac. Sci. Ibaraki Univ. Ser. A Math. 8 (1976), $1-45$.

9. N. H. McCoy, Remarks on divisors of zero, Amer. Math. Monthly 49 (1942), 286-295. MR 3, 262.

10. M. Nagata, Local rings. Interscience Tracts on Pure and Appl. Math., no. 13, Interscience, New York, 1962. MR 27 \# 5790.

11. D. Quillen, Projective modules over polynomial rings, Invent. Math. 36 (1976), 167-171. MR 55 \#337.

Department of Mathematics, Florida State University, Tallahassee, Florida 32306

Department of Mathematics, PURdue University, West Lafayette, Indiana 47907 